\title{
Expression of CD69 molecule in Rheumatoid Arthritis Iraqi patients
}

\author{
Hayfaa S. AL-Hadithi* FICM/path
}

Abstract:

Background: Rheumatoid arthritis (RA) is an autoimmune disease, where the normal joint tissues attacked by body's immune system, causing their inflammation. Cluster of Differentiation 69 (CD69) is a human transmembrane C-Type lectin protein encoded by the CD69 gene. It's expression was induced by activation (in vivo and in vitro) of T lymphocytes and Natural Killer (NK) Cells. As CD69 early activation has been implicated in the pathogenesis of some inflammatory diseases, its expression on peripheral blood T-lymphocytes must be evaluated.

Fac Med Baghdad 2015; Vol.57, No.3 Received:May,2015 Accepted:June, 2015
Objective: To evaluate the expression of CD69 on peripheral blood T-lymphocytes in RA Iraqi patients. Patients and methods: This study carried out between March 2014 and May 2014. The study involved 40 patients diagnosed with RA attending rheumatology outpatient's clinic at Baghdad medical city teaching hospital diagnosed clinically and classified according to the 2010 American College of Rheumatology (ACR) and European League Against Rheumatism (EULAR) classification criteria for rheumatoid arthritis, compared to 40 apparently healthy individuals as a control group. Erythrocyte sedimentation rate (ESR) was measured by Westergren method. Flow cytometric immunophenotype analysis applied on T-lymphocytes for measuring the fluorescence intensity of CD3 and CD69 expression on those cells.

Results: This study showed that the mean of ESR in RA patients was (66.8) while in healthy control it is only (20.4). And the mean of CD3 was higher in healthy individuals (41.88) than in RA patients (35.32), while the mean of CD69 was significantly higher among RA patients (11.09) than that of healthy controls (3.58). Also a moderately strong positive linear correlation was found between CD69 expression and RA severity. Conclusions: the expression of CD69 was up-regulated in freshly isolated peripheral blood lymphocytes from RA patients in comparison to healthy individuals.

Keywords: Rheumatoid arthritis, CD69 and Disease Activity Score (DAS28).

\section{Introduction:}

Rheumatoid arthritis (RA) is an autoimmune disease, where the normal joint tissues attacked by body's immune system, causing their inflammation (1).Although it is properly considered a disease of the joints, extra-articular manifestations can be caused by abnormal immune responses (2). In some cases, production of rheumatoid factor (RF) with the formation of immune complexes that fix complement contributes to those extra-articular findings (3).

Most people used to be treated with corticosteroids/nonsteroidal anti-inflammatory drugs (NSAIDs), and then progressed to non-biologic disease-modifying antirheumatic drugs (DMARDs) mostly if people had not responded to the previous drugs (4).

RA is the most common inflammatory arthritis, affecting $0.5 \%$ to $1 \%$ worldwide. It can occur at any age but mainly begins after age 40(5). The disorder is much more common in women (6). The exact causes of RA are unknown. But research has shown that several factors may contribute to the development of RA as genetic factors which can be triggered by environmental factors (7).

\footnotetext{
*Dept. of Microbiology \& Immunology/Collage of Medicine/ University of Baghdad .

hayfaaalhadithi@yahoo.com
}

The detailed immunogenetic studies provided clues in understanding the causes of RA (8). The traditional paradigm suggests that $\mathrm{T}$ cells in the joint activated by a pathogenic stimulus and subsequently drive an antigen-specific response. There is no requirement for a specific arthritogenic antigen to initiate the process because $\mathrm{T}$ cells with a memory phenotype accumulate in the joint owing to the release of chemoattractants. A nonspecific stimuli permits subsequent ingress of the correct $\mathrm{T}$ cell phenotype to engage resident synovial lining cells (9).

Cluster of Differentiation 69 (CD69) is a human transmembrane C-Type lectin protein encoded by the CD69 gene. It's expression was induced by activation (in vivo and in vitro) of T lymphocytes and Natural Killer (NK) Cells. While CD3 antigen used to be a useful immunohistochemical marker for T-cells in tissue section because it bounds to the membrane of all mature T-cells (10).

CD69 belongs to a family of receptors that modulate the immune response and whose genes are clustered in the natural killer gene complex, present in humans on chromosome 12. Expression of CD69 at the cell surface induced by $\mathrm{T}$ lymphocytes activation. In addition, cross-linking of CD69 by specific antibodies leads to the activation of cells bearing this receptor and to the induction of effecter functions. However, 
the physiological ligand of CD69 is unknown (11). The expression of CD69 has been studied in many autoimmune diseases as Systemic Lupus Erythematosus (SLE) and celiac disease. In earlier reports, T cells obtained from synovial fluid of patients with rheumatoid arthritis and juvenile rheumatoid arthritis, over express CD69+ antigen when compared to normal controls (12). Since the early activation antigen CD69 has been implicated in the pathogenesis of some inflammatory diseases, we evaluated the expression of this molecule on peripheral blood T-lymphocytes.

\section{Materials and method:}

This study carried out between March 2014 and May 2014. The study involved 40 patients diagnosed with RA attending rheumatology outpatient's clinic at Baghdad medical city teaching hospital, diagnosed clinically and classified according to the 2010 American College of Rheumatology (ACR) and European League Against Rheumatism (EULAR) classification criteria for rheumatoid arthritis (13), compared to 40 apparently healthy individuals as a control group. The study was approved by the ethical committee of the microbiology department in Baghdad University. The age was matched between the patients and controls groups, ranging from (3560) years.

Two milliliter Blood sample were obtained from each individual by venous puncture, then added to EDTA anticoagulant and was used for the erythrocyte sedimentation rate (ESR) and flow cytometric immunophenotype analysis. ESR was measured by Westergren method (3).

Flow cytometric immunophenotype analysis applied on T-lymphocytes for measuring the fluorescence intensity of CD3 and CD69 expression on those cells, by use of Monoclonal Antibodies Detecting Human Antigen (CD69) (Becton, Dickinson (BD) company, USA) catalogue number (341652), and Monoclonal Antibodies Detecting Human Antigen (CD3) (R\&D systems) catalogue number (FAB100F). Usually CD3 was measured routinely because it is the marker used to capture T-lymphocytes.

The results were expressed as mean and SD by using Statistical package for Social Sciences (SPSS) version 19.0. ANOVA test and ROC analysis were applied also, the value of $(p<0.001)$ was considered statistically highly significant.

\section{Results:}

Table (1) shows range, mean, SD and SE of blood ESR level, CD3 and CD69 expression among all study individuals. It shows that the level of blood ESR and CD69 were significantly higher among RA patients. This result also shown as dot diagram in figure (1).
Table (1): The case-control differences in the means of ESR, CD3 and CD69

\begin{tabular}{cccc}
\hline \multicolumn{5}{c}{ Study group } \\
\hline \multicolumn{5}{c}{ Controls } & Cases & P (t-test) \\
\hline Blood ESR & & $<0.001$ \\
\hline Range & $(8-80)$ & $(10-100)$ & \\
\hline Mean & 20.4 & 66.8 & \\
\hline SD & 16.9 & 29.7 & \\
\hline SE & 2.66 & 4.7 & \\
\hline N & 40 & 40 & \\
\hline CD3 & & & \\
\hline Range & $(4.24-62.08)$ & $(7.59-62.54)$ & \\
\hline Mean & 41.88 & 35.32 & \\
\hline SD & 16.93 & 16.95 \\
\hline SE & 2.677 & 2.68 \\
\hline N & 40 & 40 & \\
\hline CD69 & & & \\
\hline Range & $(0.97-9.42)$ & $(1.4-27.43)$ & \\
\hline Mean & 3.58 & 11.09 & \\
\hline SD & 2.14 & 5.32 \\
\hline SE & 0.339 & 0.842 \\
\hline N & 40 & 40 \\
\hline
\end{tabular}

\section{SD-Standard Deviation}

SD-Standard Error

N-total number

NS-Non Significant



Figure (1): Dot diagram with error bars showing the casecontrol difference in the median of CD3 and CD69

The RA patients were divided according to disease activity score (DAS28) by rheumatologist into three groups (mild, moderate and sever). The associations between the expression of CD3 and CD69 with disease activity were shown in figure (2), CD69 expression was significantly higher among RA patients with moderate activity. Figure (3) shows the moderately strong positive linear correlation between CD69 and RA activity score. 


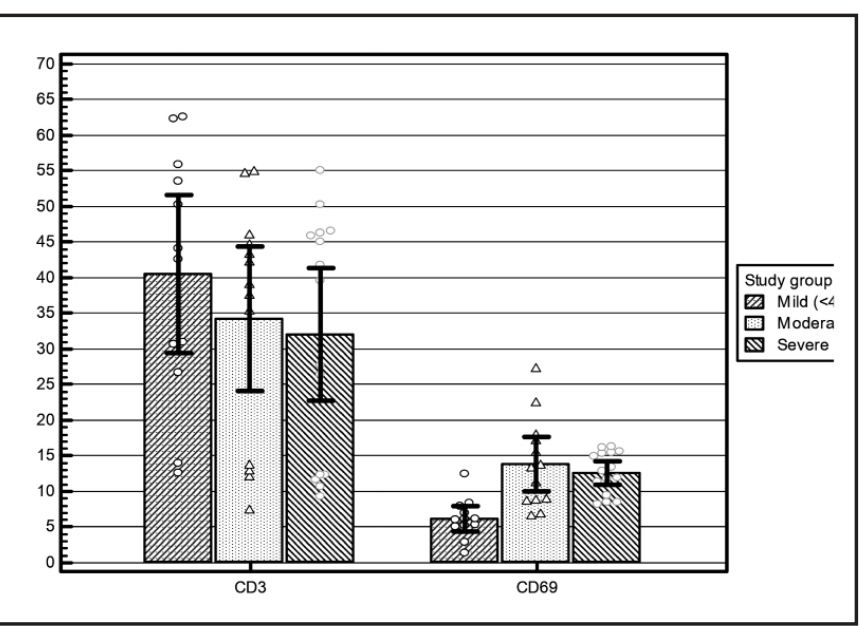

Figure (2): Dot diagram with error bars showing The association between the expression of CD3 and CD69 with (DAS28)

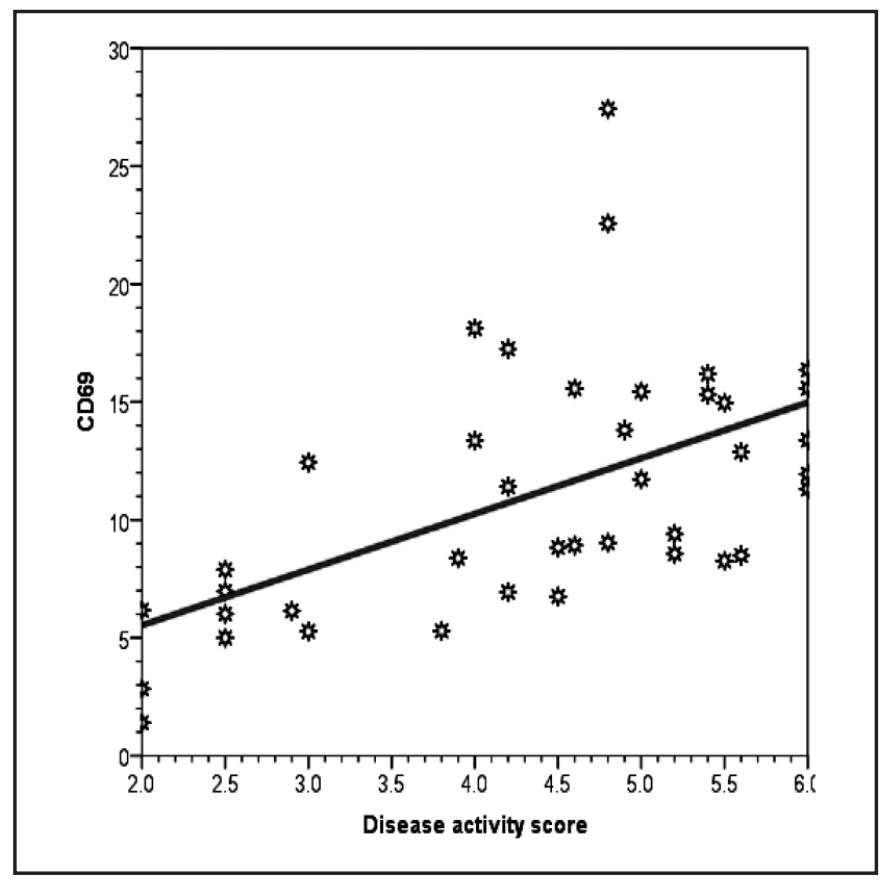

Figure (3) linear correlation between CD69 and RA activity score (DAS28).

ROC curves in figures (4) and (5) show the validity of blood ESR, CD69 and CD3 markers according to sensitivity, specificity and accuracy. This also helps in demonstrating the optimum cut-off value for each test in addition to calculation of highest specificity and highest sensitivity. Optimum cut-off for better accuracy were $(35,5.20$ and 46.57) for blood ESR, CD69 and CD3 respectively .

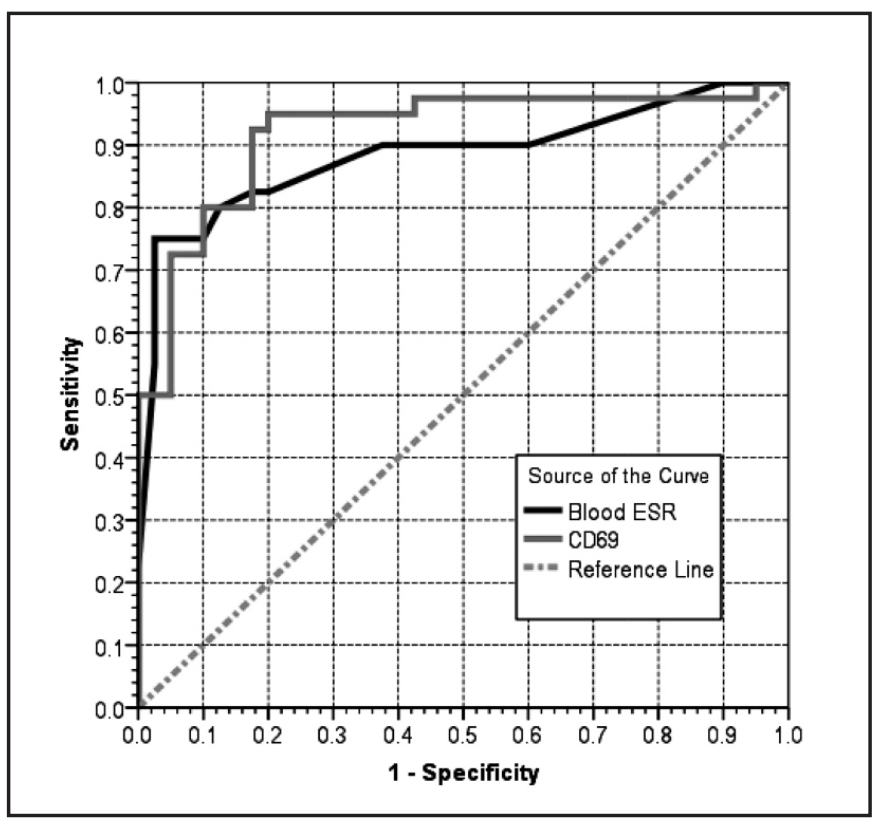

Figure (4): The validity of blood ESR and CD69 in the diagnosis of rheumatoid arthritis



Figure (5): The validity of $\mathrm{CD3}$ in the diagnosis of rheumatoid arthritis

\section{Discussion:}

As the measures of blood ESR indicate inflammation (13). In this study, RA patients have abnormally high blood ESR because RA is the most common inflammatory arthritis.

The early studies suggested an unrestricted abundance of cytokines in RA. Factors produced by macrophages and by synovial fibroblasts are markedly increased, whereas factors generated by $\mathrm{T}$ lymphocytes are low in RA (14). A non significant difference in CD3 molecule in RA patients in comparison to healthy individuals was estimated in this study. This could be due to therapeutic intervention that restore 
Treg activity which downregulate other T cells and cytokine production. Some previous studies documented that peripheral blood Treg were unable to suppress $\mathrm{T}$ cell or monocyte cytokine production in response to in vitro stimuli such as antiCD3 and anti-CD28 antibody(10). Since the up-regulation of the activation marker seems to be an indicator of the high proportion of activated lymphocytes that accumulate in the affected joints, some studies concluded the evidence of CD69 expression on synovial fluid neutrophils from RA patients (15). In agreement with previous studies, this study shows that CD69 expression was significantly higher in peripheral blood from RA patients.

Also a moderately strong positive linear correlation was found between CD69 and RA severity. This explained previously by the fact that the early activation antigen CD69 has been implicated in the pathogenesis of some inflammatory diseases and This abnormal CD69 expression could result in abnormal proliferation of autoreactive lymphocytes that perpetuate the inflammatory response(16).

\section{Conclusions:}

The expression of CD69 was up-regulated in freshly isolated peripheral blood lymphocytes from RA patients. However, the mechanism(s) of induction and its possible role in the pathogenesis of RA remain to be defined .

\section{References:-}

1. Scott D.L., Wolfe F. and Huizinga T.W.; Rheumatoid arthritis. Lancet. (2010) Sep 25;376(9746):1094-108.

2. Brooks PM.; The burden of musculoskeletal disease- a global perspective. Clin Rheumatol. (2006) Nov;25(6):77881 .

3. Saraux A., Berthelot J.M., Chal 'ES G. et al.; Value of Laboratory Tests in Early Prediction of Rheumatoid Arthritis. Arthritis \& Rheumatism (Arthritis Care \& Research), (2002). Vol. 47,(2):155-165.(IVSL)

4. Saag KG., Teng GG., Patkar NM., et al.; American College of Rheumatology recommendations for the use of nonbiologic and biologic disease-modifying antirheumatic drugs in rheumatoid arthritis. Arthritis and rheumatism. (2008) Jun 15;59(6):762-84.

5. Lawrence RC., Helmick CG., Arnett FC., et al.; Estimates of the prevalence of arthritis and selected musculoskeletal disorders in the United States. Arthritis Rheum. (1998) May;41(5):778-99.

6. Sacks JJ., Luo YH. and Helmick CG.; Prevalence of specific types of arthritis and other rheumatic conditions in the ambulatory health care system in the United States, 20012005. Arthritis Care Res (Hoboken). (2010) Apr;62(4):460-4. 7. Crowson CS., Matteson EL., Myasoedova E., et al.; The lifetime risk of adult-onset rheumatoid arthritis and other inflammatory autoimmune rheumatic diseases. Arthritis
Rheum. (2011) Mar;63(3):633-9.

8. Lessard, C.J.; Ice, J.A.; Adrianto, I. et al.; The genomics of autoimmune disease in the era of genome-wide association studies and beyond. Autoimmunity reviews. (2012) 11, 267-275

9. Liao, KP., Alfredsson, L. and Karlson, EW.; "Environmental influences on risk for rheumatoid arthritis". Current opinion in rheumatology. ( (2009) 21 (3):279-83.

10. Leong A., Cooper K. and Leong F.J.; Manual of Diagnostic Cytology (2 ed.). Greenwich Medical Media; (2003) Ltd. pp. 63-64.

11. Esplugues E., Sancho D., Vega-Ramos J. et al; Enhanced antitumor immunity in mice deficient in CD69. J Exp Med; (2003) 197:1093-1106.

12. Saber Kh, El-Khayat Z., Ramzy T. and Hanna A.N.; The Study of CD 69 as an Early Marker of SLE Activity in Pediatrics. Journal of Medical Sciences, (2007) 7: 74-80.

13. Aletaha D, Neogi T, Silman AJ, et al.; Rheumatoid arthritis classification criteria: an American College of Rheumatology/European League Against Rheumatism collaborative initiative. Arthritis and rheumatism. (2010) Sep;62(9):2569-81.

14. Rossana S., Manuela DF., Antonio S. and Guido V.; The Immunology of Rheumatoid Arthritis ; Annals of the New York Academy of Sciences (2007) ISSN: 00778923 Vol:1108 Pages: 312-322 (IVSL).

15. Atzeni, F., Del Papa N., Sarzi-Puttini P. et al; CD69 expression on neutrophils from patients with rheumatoid arthritis. Clin. Exp. Rheumatol. (2004) 22: 331-334.

16. Fernandez-Gutierrez B., Hernandez-Garcia C., Banares A.A. and Jover J.A.; Characterization and regulation of CD69 expression on rheumatoid arthritis synovial fluid $T$ cells. J. Rheumatol. (1995) 22: 413-420. 